dulsaizen durch doppelte Wahlrerwandschaft unaullösliche Niederschläge geben, mit letzleren, wenn sie mit Stickoxyd gesältigt sind, Niederschläge geben, welche aufgenommenes Gas gebunden enthalten; allein diese Verbindungen sind so wenig beständig, dafs man ihre Eigenschaften nicht studiren kann.

Es ist bemerkenswerth, dafs das in diese unbeständigen Verbindungen eingehende Stickoxyd genau so viel Sauèrstoft enthält als nöthig wäre, um das in der Verbindung enthaltene Eisenoxydu! in Oxyd zu verwandeln. Herr Peligot gründet auf diese Eeobachtung die Meinung, als sey das Stichoxyd mit dem Eisenoxydul als solchem verbunden und als spielten diese beiden Oxyde mit cinander vereinigt die Rolle einer einzigen Base.

\title{
Ueber einige neue Produkte, welche sich durch die Finwirkung der Alkalien anf fette Körper bei hoher 'I'emperatur bilden
}

von

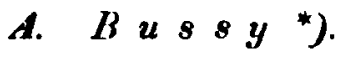

(Annales de chimie et de physique, Aout 1833.)

Im Verlauf der organisch chemischen Untersuchungen bieten sich ziemlich häufig neue Ilörper der Aufmerksamkeit des Beobachters dar. Wollte derselbe sich aber nur darauf beschränken, deren Eigenschaften auszumitteln und deren wahrscheinliche Anwendbarkeit anzugeben, so würde er blos

*) Wir geben diese schöne Arbeit vollstïndig, indem sie sich an die Versuche über das Benrol anreiht und gleichsam eine Portsetzung davon billet.

D. $\mathbf{h}$. 
die Hälfte sciner Aafgabe gelöst und der Wissenschaft darch Anhäufen neuer Produlte keinea grofsen Gewinn gebracht habea. Zur Förderung der Wissenschaft ist hauptsächlich nothwendig, dafs man die Realtionen studirt, mittelst welcher die organischen Produlte sich eins in das andere verwandeln, die Verhältnisse feststellt, in welchen sie durch ihre Zusammensetzung zu einander stehen und die Verbältnisse der Abstammung zwischen dem Hauptprodult und den daraus entstandenen ausmittelt, damit man leicht das Gesetz dieser Modifikationen übersehen und sie anf eine systematische Weise ordnen trann.

Hauptsächlich von diesem Gesichtspuntte ausgehend habe ich die jetzt zu beschreibenden neuen Produlte studirt, in der Veberzeugung, dafs uns die Elemente eines guten Systems der organischen Chemic noch fehlen und dafs die Fortschritte in diesem Theil der Wissenschaft für den Augenblick von der Beharrlichkeit der Chemiker und der Vervielfachung der Untersuchungen abhängen.

Schon vor einigen Jahren haben Hr. Le Canu und ich gezeigt, dafs man beim Erhitzen gewisser fetter Körper, besonders thierischen Ursprungs, als Zersetzangs - Prodult derselben ein Gemisch von Oelsäure, Margarinsäure nebst andern Pyrogensubstanzen erhalte; allein dies verhält sich nicbt mehr also, wenn man, anstatt die fetten Materien fiusich allein zu destilliren, sie mit Kalk, Baryt, Strontion, Kali und Natron erhitzt.

Destillirt man z. B. 100 Gr. Talg aus einer Glasretorte mit der Hälfte seines Gewichts Aetzkalk und fingt man die Produlte separirt auf, so wird man zuerst eine flüssige, beim Erlialten fest werdende Masse erhalten, welche im Anfang der Operation fast farblos, wach und nach immer gelber wird, ihre Consistenz verliert und am Ende der Operation ganz flüssig erhalten wird. 
Presst man dec festen oder weichen Theil des Desiillars zwischen Fliefspapier aus, so absorbirt dasselbe eine gelbe empyreumatische ölige Materie, und es bleibt eine feste glänzende perlmutterartige leicht gelbliche Materis zurüch, welche man leicht durch Behandlung mit siedendem Alkohol reinigen hann, da sie sich in der Hitze in demselben auflüst und beim Erikalten größstentheils wieder ausscheidet. Diese Materie besitzt im Aeufsern einige Aehnlichkeit mit der Margarinsäure, allein sie unterscheidet sich von derselben durch sehr bestimmte Eigenschaften, unter andern durch die $\mathbf{A b}$ wesenheit aller sauren Eigensehaften und durch Unverseifbarkeit mit den Aetzalkalien, sowohl in der Hitze als in der Kälte.

Diese neue Materie, welche aufserdem noch einige merkwürdige Eigenschaften besitzt, schien mir hinsichtlich ihrer Zusammensetzung und der Analogie, welche sie mit den fetten IGörpern, aus denen sie entstand, zeigen kann, eine genaue Untersuchung zu verdienen. Allein um diese mit Vortheil anstellen zu können, mufste ich zuerst die Bedingungen zu ihrer Darstellung zu vereinfachen suchen.

Die fetten Körper hesitzen, wie man seit dea schönen Arbeiten Chevreuls weifs, eine sehr verwickelte Zasammensetzung und rerändern sich durch den Einflufs der Alkalicn leicht in andere Hörper saurer Natur; es war demnach ziemlich natürlich, die von der Destillation des Talgs mit dem Kalk erhaltenen Materien für das Prodult einer secandairen Wirkurg des Kalks auf die früber von demselben bei einer niedrigeren Temperatur erzeugten fetten Säuren $z u$ halten. Diese Annahme wurde durch das Experiment als wahr befunden und erlaubte mir sowohl die Operation zu rereinfaehen, als auch die Theorie derselben besser anszumitteln. Ich habe daher Margarinsäure, Talgsäure und Oelsäure jede für sich allein mit Kalk destillirt und von jeder derselben 
sigeathümliche Materien erhalten, ron donen ich nun besonc.ers handein will.

Margariusäure.

Dis Margarinsäure, deren ich mich bediente, ward durch Destillation von 'Talg erhalten und durch Auspressen des Produbts und Umbrystallisiren aus Alhohol gereinigt; aie schmole bei $56^{\circ}$. Ich gab dieser Säure ror der durch Saponilkation erbsitenen den Vorzug, weil sie heine Talgsëure enthält und von den anbängenden flüssigen Produlten leicht gereinigt werdea hann.

Mit dom 4ten Theil ibres Gewichts Aetzkalk gemischt und aus einer Retorte destillirt geht zuerst eine geringe Quantitït Wasser, alsdann eine weiche Masse, aus welcher man duncb Auspreasen eiae der aus Talg erhaltenen ähnliche Materie gewinnt. Die letzten Portionen der Säure erleiden eine vollkommnere Zersetzung, denn das am Ende der Destillation Uebergehende ist gefärbt und empyreumatisch; in der Retorte bleibt Aetzkalk, Hohlensaurer Halk und eine tleine Menge Kohle zurück, welche die Masse schwarz färbt. 40 $\mathrm{Grm}$. Margarinsüure gaben also behandelt $28 \mathrm{Grm}$. festen leicht gelben, Fettflecken auf dem Papier machenden Produkts, aus welchem $22 \mathrm{Grm}$. trockene Materie erbalten wurden; diese letztere schmolz, nachdem sio von allen flüssigen Produkten befreit worden, bei $74^{\circ}$. Sie ward mehrmals mit siedendem Alkohol ron $36^{\circ}$ behandelt und nachdem dies in mal geschehen, war der Schmelzpunht der letzten Portion auf $77^{\circ}$ gestiegen und blieb von da an beständig. Ich erhielt gleiche Resultate bei der Destillation von margarinsuurem Kalk, der durch Auflösen der Säure in schwacher Aetzlauge und Niederschlagen derselben mit Chlorcalcium erhalten vo:den war.

Die unter diesen rerschiedenen Umständen erhaltene Materie ist, aus Altohol krystallisirt, rein weifs, glänzend, 
perlmutterartig, sohmilzt wie schon angegeben bei $77^{\circ}$, krystallisirt beim Erkalten verworren und sieht alsdanu wie Margarinsäure oder Wallrath aus. Sie leitet nicht die Elelstricität, sondern wird durch Reilong ou!er Druck stark elelitrisch, so dafs sie in einem Achatmörser gerieben an den Winden desselben und an dem Pistill binaufsteigt und an dem Papier anhängt, womit man sie umrührt. In einer Retorte erhitzt geräth sie in Sieden und destillint ohne besondere Zersetzung und ohne einer Riichstand zu lassen, über. In hoher Temperatur brennt sie mit sehr glänzender Flamme obne Rauch, ebenso brennt sie am Docht. In siedendem Alkonol von $36^{\circ}$ ist sie löslich, allein in viel geringerer Quantitüt als die Morgariosïure; 1 Gr. löste sich vollständig in $50 \mathrm{Gr}$. Sie vertheilte sich dabei zuerst in hleine am Boden liegende Kügelchen, die sich erst beim Schütteln lösien. Beim Erkalten der Lösung, wie anch bei Zusatz von $W$ asser scheidet sie sich gröfstentheils wieder aus. In Alkohol von $40^{\circ}$ löst sie sich im Verhältnifs von $3 \mathrm{zu} 20$ und die Lösung erstarrt beim Erkalten 2n einer Masse. Schwefeläther löst in der Hitze mehr als $1 / 5$ seines Gewichts, wovon der gröfste Theil beim Erkalten wieder niederfällt, ebenso Essigäther und Terpentinöl. Mit Phosphor läfst sie sich beim Schmelzen nicht mischen, allein sie löst eine gewisse Menge desselben auf. Mit Kampfer verbindet sie sich in allen Verhältnissen. Durch siedende concentrirte Aetzkalilösung wird sie nicht verändert. Schwefelsäure färbt sie und zerlegt sie zuletzt gänzlich unter Entwicklung von schweflichter Säure. 1 Gr. mit 2 Gr. Schwefelsäure gelinde erhitzt färbte sich anfangs roth, dann braun, zuletzt dunkelschwarz und nach einigen Augenblicken war alles unter reichlicher Entwicklung ron schweflichter Säure und unter bleinen Detonationen in eine lohlige Masse verwandelt. Salpetersäure greift sie nur in der Wärme sehr schwach an. In einer Röhre bei gelin- 
der Wärme mit Chlor behandelt wird sie vollständig in eine farblose durchsichtige flüssige, bei gewöhnlicher Temperatur klebrige Materie verwandelt.

Diese Materie, welche ich mit dem Namen Margaron bezeichnet habe, besitzt hinsichtlich ihrer Eigenschaften einige Aehnlichleit mit Hrn. Reichenbachs Paraffin und nähert sich demselben auch in seiner Zusammensetzung, wie man sogleich sehen wird; sie unterscheidet sich von demselben jedoch wesentlich durch ihren Schmelzpunlt, welcher bei $77^{\wedge}$ liegt, wïhrend das Paraffin schon bei $43 \frac{3}{4}$ schmilzt, wie auch durch ibr Verhalten gegen Schwefelsäure, von weicher das Paraffin nicht zersetzt wird.

Zusammensetzung :

Die Analyse des Margarons ward mittelst des Liebigschen $\Delta$ pparats ausgeführt und in 5 Anal. wurden erhalten:

I. II. III. IV. V.

Kohlensäure $\quad 1,51 \quad 1,51 \quad 1,505 \quad 1,504 \quad 1,506$

$\begin{array}{llllll}\text { Wasser } & 0,609 & 0,612 & 0,606 & 0,610 & 0,602\end{array}$

Im Mittel waren nach diesen Analysen 1,507 Koblensäure und $0,608 \mathrm{~W}$ asser erhalten worden und die Zusammensetzung wäre demnach in 200 Theilen:

$\begin{array}{lr}\text { Kohlenstoff } & \mathbf{8 3 , 3 4} \\ \text { Wasserstoff } & 13,5: \\ \text { Sauerstoff } & \mathbf{3 , 1 5}\end{array}$

Man sieht aus dieser Zusammensetzung, dafs sich die analysirte Materie sehr dem Koblenwasserstoff nähert. Die darin enthaltene Quantität Sauerstoff ist sebr gering und lann hinsichtlich ihrer Zusammensetzung in Atomen nur in sehr geringem Atomverhältnif darin enthalien seyn. Um übrigens leinen Zweifel über die Gegenwart des Sauerstoffs zu lassen, behandelte ich die geschmolzene Substanz mit Kalium, welches sich damit zersetzte und eine geringe Mlenge brennbaren Gases erzeugte. Es ist kaum nöthig zu erinnern, 
daft die Substanz lange Zeit bei $130^{\circ}$ im Flufs erhalten warden war, um sie so vollstöndig als möglich auszutrockne.r.

Es ist sehr schwierig aus dieser Analyse irgend eine Formel herzule:ten, denn nähme man auch nur a At. Sauerstoff ar, so würde man mehri als 70 Atome Wasserstoff erhalten und die Grenzen der unvermeidlichen Fehler in der Bestimmung des Wasserstoffs crlauben nicht das geringste Vertrauen zou den Atomzahlen, welche die Mechnung gibt. Hauptsächlich in ähnlichen Fiallen ist es wichtig, die durch den Versuch gefundene Zusammensetzung von einem theoretischen Gesichtspunbte aus zu betrachten, welcher die Resultate $\mathrm{zu}$ beurtheilen und die unvermeidlichen Irrthïmer $\mathrm{zu}$ verbessern erlaubt.

Ich versuchte in dieser Absicht zuerst dus sperifische Gewicht des Margarondampfs nach Hr. Dum as Methode zu nehmen, mufste aber dieses Mittel aufgeben, da cine beträchtliche Menge vor der vollständigen Verfüchtigung zersetzt ward.

Vergleicht man jedoch die gefundene Znsammensetzang mil der der Margarinsäure selbst, so findet man sehr merkwürdige Verhältnisse zwischen denselben. Die Margarinsäure besteht nach Hr. Chevreul aus:

$\begin{array}{lrl}\text { Kohlenstoff } & 79,053 & \mathrm{C}^{35} \\ \text { Wasserstoff } & 12,010 & \mathrm{H}^{65} \\ \text { Sauerstoff } & 8,037 & \mathrm{O}^{3}\end{array}$

die Talgsäure nach cben demselben aus:

$\begin{array}{lrl}\text { Kohlenstoff } & 80,145 & C^{30} \\ \text { Wasserstoff } & 13,47^{8} & \mathbf{H}^{134} \\ \text { Sauerstoff } & 7,377 & 0^{5}\end{array}$

allein aus Gründen, die mir selır wichtig scheinen, hält Hr. Berzelius $\mathrm{C}^{35} \mathrm{H}^{67} \mathrm{O}^{3}$ für die wabre Formel der Margarinsäure; so dals wenn man $\mathrm{C}^{45} \mathrm{H}^{67}$ mit $\mathrm{A}$ bezeichnete, die beiden in Rede stehenden Säuren ein und dasselbe hadi- 
kal hätten und die eine mit $\mathrm{A} \mathrm{O}^{3}$, die andere mit $\mathbf{A}^{2} \mathrm{O}^{5}$ Bexeichnet worden könnten. Der ganze Unterschied hängt, wie man sieht, ron 2 At. Wasserstoff mehr oder weniger $a b$, und wenn auch die so wohl behannte Genanigkeit des Analytikers diese Veränderung nicht zu erlauben scheint, to kann sie woht einigermafsen durch die Schwierigkeit, mit der die von ihm untersuchten Produkte rein darzustellen sind, entschuldigt werden*).

Sey dem wie ihm wolle, so sieht man dafs sich, unter Annohme der Formel $\mathrm{C}^{35} \mathrm{O}^{3} \mathrm{H}^{67}$ für die Margarinsäure, dieselbe durch $\mathrm{CO}:+\mathrm{OH}^{67} \mathrm{C}^{54}$ ausdrüchen lärst. Die Formel $\mathrm{OH}^{6:} \mathrm{C}^{31} \mathrm{~g}^{\mathrm{ibt}}$ in 100 Theilen :

$\begin{array}{lr}\mathrm{C} & 83,38 \\ \mathrm{H} & 18,41 \\ \mathrm{O} & 3,21\end{array}$

Die analysirte Materie A gibt:

$\begin{array}{lr}\text { C } & 83,34 \\ \text { H } & 13,51 \\ \text { O } & 3,15\end{array}$

*) Diese Zusammensetzungsverhältnisse waren Hrn. Cherreul sclbst nicht entgangen, da er fiir die Talgsäure den Namen Margarichter Säurc vorschlug. Diese Neuerung wäre zu der damaligen Zeit zu gewagt gewesen, würde aber jetzt durch die neueren Entdeckungen in der organischen Chemie lrin!änglich gerechtfertigt scyn. Ich mufs noch hinzufügen, dals Hr. Cbev reul in seiner ersten Abhandlung uber die fetten Körper bei Gelegenheit der Destillation der Margarinsäıre angibt, dafs er unter den nicht verseifbaren Produkten dieser Destil. lation eine feste perlmutterartige Substanz lemerlt habe, welche viel Analogie wit der Margarinsäure besitze. Es wäre möglich, dafs diese Materie nichts anders als Margaron var. Es ist dies eine näher zu untersuchende Sache, deren Erklä. rung sirh aber leicht aus der von mir gegebenen Theorie folgern liefse.

**) Die Resultace dieser Analyse fullen fast mit denen zusammen, dic Hr. Pelletier von dem Amberfett erhielt. (Annales de chim. et de phỳs II 51 - Diese Annaicn Bd. VI S. 25.) 
Diesp Zahlen stimmen beinahe vollkommen mit den ersten überein, die Zusammensetzung des Margarons kann demnach durch $O{ }^{16}{ }^{67} \mathrm{C}^{34}$ ausgedrückt werden und das Margaron wäre daher nichts anders als Margarinsüure weniger $1 \mathrm{dt}$. Hohlensäure, denn $\mathrm{O}^{\mathbf{4}}{ }^{67} \mathrm{C}^{34}=\mathrm{O}^{3} \mathrm{H}^{67} \mathrm{C}^{35}-\mathrm{CO}^{2}$. Um diese Zusammensctzung durch einen andern Versuch zu bestätigen, mischte ich \& At. Var garinsäure (das in der lirystallisirten Säure enthaltene Wasser in Kechnung gebracht) mit 1 At. Aetzbaryt sehr genau zusammen und erhielt in der That dieselbe Materie als Prodult und A At. durch etwas Kohle geschwärzten kohlensauren Baryt. Multiplicirt man die Atomzablen der destillirten Materie mit 3 , so erhäit man $\mathrm{O}^{3} \mathrm{H}^{201} \mathrm{C}^{: 02}=\mathrm{O}^{3} \mathrm{H}^{67} \mathrm{C}^{15}+\mathrm{H}^{134} \mathrm{C}^{67}, \mathrm{~d}$. h. sie lälst sich ausdrücken durch Margarinsäure nebst doppelt Kohlenwasserstoff. In diesem Falle wäre sie, wie man sicht, eine Art Margarin-Aether, in welchem die Eigenschaften der Säure durch den Kohlenwasserstoff neutralisirt sind.

In dieser Hinsicht bietet diese Materie cine überraschende Analogie mit dem Pyro-Essiggeist dar, welcher nach den Analysen der HHrn.Liebig und Dumas als Essigsäure mit Kohlenwasserstoff - Hydrat betrachtet werden liann*). Das Margaron, dessen Formel $\mathrm{C}^{34} \mathrm{H}^{17} \mathrm{O}$ oder $\mathrm{C}^{18} \mathrm{H}^{134} \mathrm{O}^{\text {: }}$ ist,

") In der Abhandlung, welche ich der Alademie überreichte, latte ich den Namen Pyro-Margaringeist angenommen, wegen uer Analogie der Zusammensetıung dieser Materie nit dem Essiggeist; allein nach den richtigen Bemerkungen der Berichtcrstatter über meine Arbeit, HHrn. Thenard u. Chevreul, mulste ich diese Benennung ändern. Ich habe dafür Margaron gewählt, substantivum femininum ron Margarique. Diese Benennungsweise erleichtert die Bildung zusammengesetzter Namen und exinuert zugleich an die Stammsubstanz. Nach die.sem Nomenklatur - Princip nüfsten der Pyroessiggeist, Talg und Oelgeist durch Acèton, Stéaron und Oléon bezeiclinet werden. 
Kann selbst als Kohlenwasserstoff mit Kohlensäure betrachtet werden, weil $\mathrm{C}^{68} \mathrm{H}^{134} \mathrm{O}^{2}=\mathrm{CO}^{2} \mathrm{C}^{6 i} \mathrm{H}^{134}$; es war demnach sehr natïrlich zu schliefsen, dafs wenn man dem Margaron durch Behandlung mit Aetzalkalien in hoher Temperatur das $1 / 2$ At. Kohlensäure welches es enthält, entziehen künne, man Paraffin orhalten müsse. Dies geschieht in der That, allein auf unvollkommne Weise, weil einerseits heine Verbindung des Margarons mit dem Alkali bei niedriger Temperatur erfolgt und andrerseits bein Erhitzen des Gemisches sich das Margaron vermöge seiner Flüchtiglieit gröfstentheils der Einwirbung des Allialis entzieht. Nichtsdestoweniger erhält man durch Destillation des Margarons mit der Hälfte seines Gewichts Aetzkalk eine Materie, deren Schmelzpunlt bei ungefähr $60^{\circ}$ liegt und deren Eigenschaften sich denen des Paraffins nähern, während der Rückstand eine gewisse Quantiät liohlensauren Kalk enthält. Ich zweifle nicht, dafs man durch öftere Wiederholung dieser Behandlung auf geeignete Weise das Margaron vollständig in Paraffin verwandeln kann. Die Margarinsïure kann man sich demnach in ihren Elementen als Kohlensäure mit Doppelt-Kohlenwasserstoff vorstellen, welche durch Entziehung von $2 / 3$ ibrer Kohlensäure durch Destillation mit Alkalien in Margaron und durch Entziehung des letzten Drittels Koblensäuro in Paraffin verwandelt wird.

\section{Talgs äure.}

Nachdem ich die Wirkung des Kalks auf die Margarinsäure untersucht hatte, ward ich durch die Analogie bewogen, die andern Fettsäuren aus demselben Gesichtspunkte zu untersuchen.

Behandelt man die Talgsäure auf dieselbe Weise wie die Margarinsäure, so crhält man ebenfalls eine der ersteren sehr analoge Materie, die aber weniger leicht schmelzbar ist 
und oine etwas abweichende Zusammensetzong hat. Sie ist in 100 Theilen:

$$
\begin{array}{ll}
\text { C } & 84,7^{8} \\
\text { H } & 13,77 \\
0 & 1,45
\end{array}
$$

Naeh den Analysen des Hrn. Cherreul ist a At. Talgsö̀re $=O^{5}$ H $^{134} \mathrm{C}^{70}$, welches 2 At. Basis sättigt. Scheidet man die zur Sättigung von 2 At. Basis nöthige Menge Kohleasäure $a b$, welche bei der Destillation der Talgsäure mit den Alkalie: in der Retorte als Carbonat zurückbleibt, so hat man $\mathrm{O}^{5} \mathrm{H}^{144} \mathrm{C}^{70}=\mathrm{O}^{4} \mathrm{C}^{2}+\mathrm{H}^{131} \mathrm{C}^{68} \mathrm{O}$. Dies gibt auf 100 Theilo berechnet:

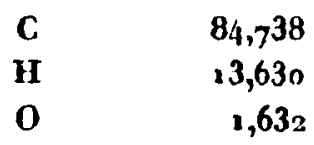

was beinahe genau mit der Zasammensetzung (C) übereinstimmt.

In diesem Falle wäre die mit dem Namen Stearon bezeichnete Materie ebenfalls eine Art Aether $\left(\mathrm{O} \mathrm{H}^{13} \mathrm{C}^{1 / 5}\right)$, dessen Formel mit 5 multiplicirt $=0^{5} \mathrm{H}^{670} \mathrm{C}^{340}=$ $\mathrm{O}^{5} \mathrm{H}^{134} \mathrm{C}^{70}+\mathrm{H}^{5,6} \mathrm{C}^{270}$, d. h. Talgsäure und Hoblenwasserstoff. Allein hier fände sich der Kohlenstoff and Wasserstoff nicht in demselben Verhältnisse wie im Doppelt Hohlenwasserstoff. Drückt man $\mathrm{H}^{67} \mathrm{C}^{34}$ durch $\mathrm{K}$ aus, so würe das Margaron $\mathrm{OK}$ und das Stearon $\mathrm{OK}$ :

Das Stearon hat die meisteı äulseren Eigenschaften mit dem Margaron gemein. Durch Krystallisation aus Alkuhol gereinigt schmilzt es bei $86^{\circ}$, und ist weniger löslich in Alkohol und Aether. Das Stearon und Margaron könnten wegen ihrer Leichtverbrennbarkeit und ihrer Eigenschaft, erst in hober Temperatur zu schmelzen, sehr gut das Wachs und andere Brennmaterialien bei Pracht-Beleuchtungen ersetzen, allein sie haben mit der Margarinsäure und Talgåăure 
die Unannehmliehseit gemein, im geschmolzenen Zustande aufserst düanflüssig zu seyn.

$$
\text { Oelsäure. }
$$

Hehandel man die Oelsäure wie die beiden rorbergehenden, so erhält man'als Hückstand ebenfalls hoklensnuren Kalk, allein als Destillationsprodukt selbst gleich ron Anfang der Operation eine flüssige Materie, welche nur Spuren fester Materien absetzt. Diese Materie ist nicht sauer, unverseifbar und scheint sich zur Oelsäure ebenso zn rerhalten, wie das Margaron und Stearon zu den beiden vorhergehenden Säuren. Die Schwierigheit, die Oelsăure rein darzustellen and das Oleon von den andern Flüssigkeiten, die sich bei der Destillation bilden lönnen, zu trennen, haben mich bis jetzt abgebalten scine Zusammensetzung und sein Verbsltnifs zur Oelsäure durch den Versuch auszamitteln.

Wenn es aber erloubt ist, sich schon jetzt eine Ansicht über dis Znsammensetzung dieses Prodults zu bilden, $\infty$ ist man durch die Analogie berechtigt, es als Oelsziure minus Ioblensäure zu betrachten, und nimmt man nach Hrn. Chevreul für die Zusammensetzung der Oelsäure die Formel $C^{70} H^{120} O^{i}$ an, so wäre die des Oleous $\mathrm{C}^{58} \mathrm{H}^{120} \mathrm{O}=$ $\mathrm{C}^{10} \mathrm{H}^{120} \mathrm{O}^{5}-\mathrm{C}^{2} \mathrm{O}^{4}$.

\section{Ueber die Theorie der Pyrogensäuren}

$$
\text { Pelo } \boldsymbol{o}^{\text {von }}=0 .
$$

(Aus einem Schreiben an J. L.)

Ich glaube die wahre Theorie der Pyrogensäuren gefun. den $z a$ haben und bin hierauf durch die Versache vicler Cherniker, so wie auch durch einige von mir selbst ange- 